Eidg. Technische Hochschule, Zürich - Fortbildungskurs der EAWAG, I964

\title{
Die Nährstoffelimination aus der Sicht des Limnologen
}

\author{
Von Dr. H. AмвÜHL, EAWAG, Zürich
}

\section{Einleitung}

In den vorangehenden Referaten ist es deutlich geworden, dass die Elimination der Pflanzennährstoffe aus dem Abwasser nicht etwa einem Perfektionsbestreben der Abwasserfachleute entspringt, sondern dass sie tatsächlich notwendig ist, eine Erkenntnis, die sich aus Beobachtungen in der Natur herleitet. Diese Beobachtungen beziehen sich auf die Verunreinigung von Seen und von stehenden Gewässern im allgemeinen.

\section{I.I Der Begriff des sauberen Sees}

Das, was wir als sauberen See bezeichnen, also ein See mit blankem Ufer, mit klarem, blauem Wasser, ist, physiologisch gesehen, ein näbrstoffarmer See. Sein Wasser enthält so wenig von jeden Stoffen, welche die pflanzliche Zelle zu ihrem Aufbau braucht, dass sich nur sehr wenige pflanzliche Organismen erhalten können, und damit auch nur wenige Kleintiere und Fische. Der See befindet sich gewissermassen in einem Hungerzustand. Bei näherer Prüfung wird er freilich von den meisten zum Pflanzenleben nötigen Nährstoffen sogar im Übermass enthalten, aber mindestens einer davon wird fehlen oder im Augenblick aufgezehrt sein. Das Wachstum der pflanzlichen Zelle ist aber nur möglich, wenn sämtliche Nährstoffe $(\mathrm{C}, \mathrm{O}, \mathrm{H}, \mathrm{N}, \mathrm{S}, \mathrm{P})$ vorhanden sind - fehlt ein einziger, so hört es auf; die Pflanze hungert. Dieser fehlende oder aufgebrauchte Nährstoff ist, wenn wir von den Spurenstoffen zunächst absehen wollen, entweder Stickstoff oder, wie es in unseren Seen meistens der Fall ist, Phosphor. 
I.2 Fliessend'es und stehendes Wasser - zwei verschiedenartige Lebensräume

Dass sich stehende und fliessende Gewässer ganz erheblich voneinander unterscheiden, macht sich nicht nur äusserlich geltend, sondern vor allem im Organismenbestand. Das Leben im Fluss und Bach ist getragen durch Makroorganismen, Würmer, Schnecken, Insektenlarven in manchen Arten und mannigfaltigster Anpassung, durch Moose, fädige Algen, Bakterien und Pilze, während das Leben im See sein Gesicht durch die mikroskopischen, einzelligen Algen erhält und die Tiere mengenmässig eher zurücktreten. Der See ist ein Ort der Produktion organischer Substanz; das Fliessgewässer ein Ort des Konsums. Im See leben, mit Ausnahme der Uferzone, völlig andere Organismen als im fliessenden Wasser; diese leben auch unter völlig anderen äusseren Bedingungen und reagieren anders auf externe Einflüsse.

Ein Wasser, das chemisch so beschaffen ist, dass es - als Bach - als recht sauber zu gelten hätte, könnte - als See oder Teich - durchaus eutrophen Charakter besitzen, weil nämlich die darin enthaltenen Pflanzennährstoffe im Fliessgewässer nicht in der gleichen Weise zur Wirkung kommen wie im stehenden Gewässer, wo sie von den Mikroorganismen aufgenommen werden. Zwischen stehenden und fliessenden Gewässern bestehen somit, ohne dies weiter auszuführen, grosse, grundsätzliche Unterschiede.

Zwar ist der von den Trägern der Urproduktion belebte Raum eines Sees, d. h. die einige Meter mächtige oberste Wasserschicht, auch nur ausnahmsweise völlig in Ruhe, meistens ist sie durch Thermokonvention und windbedingte Strömungen langsam bewegt, doch niemals im selben Masse wie ein Fliessgewässer. Während sich dort nur robuste, dem Leben im rauhen Klima des strömenden Wassers angepasste Formen zu halten vermögen, sind die Organismen des freien Sees grazil gebaut, zu einem guten Teil mikroskopisch fein und allesamt auf ein Dasein in dauernder Schwebe eingerichtet; sie werden im Begriff des «Planktons » zusammengefasst. Die wenigsten Plankter können sich aktiv fortbewegen; sie halten sich passiv in demjenigen Wasserkörper auf, in den hinein sie die Natur gestellt hat. Demzufolge wird auch keine Nahrung an sie herangetragen, sondern sie sind auf das angewiesen, was ihre unmittelbare Umgebung enthält; einzig das Licht, das zur Assimilation nötig ist, kommt von aussen in das sonst ziemlich geschlossene System hinein. Im Gegensatz zu den Organismen des Fliesswassers, welche von dem leben, was ihnen durch die fliessende Welle dauernd neu zugeführt wird, leben die Plankter somit von den Nährstoffen 
ihrer Umgebung, sind nun aber in der Lage, diese Nährstoffe auch dann noch dem Wasser zu entziehen, wenn sie nur noch in unwahrscheinlicher Verdünnung vorliegen. Diese Fähigkeit ist für das gesamte Geschehen im See von grösster Bedeutung.

\section{Der Phosphor als Nährstoff im See}

\section{I Der Stoffkreislauf des Sees}

Der limnische Stoff kreislauf spielt sich, auf den einfachsten Nenner gebracht, folgendermassen ab: In der durchlichteten und damit dem pflanzlichen Leben zugänglichen obersten Wasserschicht, der sog. trophogenen Schicht, bildet sich die als Plankton bezeichnete Lebensgemeinschaft aus Bakterien, Blau-, Kiesel- und Grünalgen, Rädertieren, Kleinkrebschen usw., und zwar je nach der Konzentration der vorhandenen Nährstoffe mehr oder weniger dicht. Diese Produktion, welche das ganze Jahr über anhält und keine eigentliche Ruhepause kennt, geht so lange weiter, bis einer der Nährstoffe völlig aufgebraucht ist. Die gebildeten Organismen haben zumeist eine kurze Lebensdauer, im allgemeinen nur einige Wochen, sterben dann $a b$ und sinken in die Tiefe. Bereits während des Absinkens setzen autolytische und bakterielle Prozesse ein, welche die Organismen teils gar nicht bis zum Grund gelangen lassen, sondern sie vorher bereits auflösen, zum andern Teil sinken sie bis zum Grund und werden dort zersetzt. Im Lauf der sommerlichen Stagnationszeit häufen sich auf diese Weise die Endprodukte des Abbaus in der Tiefe an; in entsprechenden Wasseranalysen stellen wir fest, dass sowohl Stickstoff als auch namentlich Phosphor in der Tiefe angereichert wird. Gleichzeitig bleiben aber die Folgeerscheinungen nicht aus: Der Abbau erfordert Sauerstoff, was zum bekannten Sauerstoffschwund in der Tiefe sowie in der ersten Abbauzone unterhalb der trophogenen Schicht führt. Der Sauerstoffschwund kann zur völligen Sauerstofflosigkeit werden und auf der Stufenleiter der Reduktionsprozesse noch weiter gehen bis zur Ausbildung eines Fäulnismilieus, was wiederum besondere Konsequenzen zur Folge hat: Nach den Untersuchungen von EINSELE [8, 9], OHLE [20], Thomas [32] und anderen Autoren soll Phosphat, in der Seetiefe angereichert, bei Anwesenheit von Sauerstoff in schwerlöslicher Form, z. B. als Eisen- oder Manganphosphat, teilweise im Sediment festgelegt und damit dem weiteren Stoffkreislauf entzogen werden. Tritt nun Anaerobiose auf, so wird ein Teil davon infolge Reduktion wieder löslich und tritt ins Wasser zurück. Nach experimentellen Untersuchungen 
von THOMAS [32] und von BACHOFEN [4] verlaufen diese Vorgänge in unseren Seen indessen nicht quantitativ, sondern nur zu einem unbestimmten Bruchteil. Die Stoffrückwanderung findet sowohl unter anaeroben wie auch unter aeroben Verhältnissen statt, doch wird sie durch anaerobe Verhältnisse zumindest begünstigt.

Intensität und Ausmass aller dieser Vorgänge hängen davon $a b$, welche Nährstoffkonzentrationen im See zur Verfügung stehen, z. B. am Anfang des Seejahres während der Frühjahrsvollzirkulation.

\subsection{Analytische Schwierigkeiten der Phosphorbestimmung}

So weit die Theorie und die Ergebnisse friherer Arbeiten. Es mag in der Natur der Sache liegen, dass wir heute vor allem dort über die Nährstoffverhältnisse Bescheid wissen, wo sich auffällige Änderungen vollzogen haben, also in Seen, die im Lauf der letzten Jahre oder Jahrzehnte ihr Gesicht wesentlich veränderten oder dazu als Vergleich herangezogen wurden, z. B. der Zürichsee $[19,29,30,33,34,4 \mathrm{I}]$, der Pfäffikersee, Greifensee und Ägerisee [32], der Hallwiler- und Baldeggersee [3, 4], der Untersee und andere Seen mehr. Zumeist sind es Seen, welche ziemlich hohe Nährstoffgehalte besitzen. Demgegenüber wird man in der Literatur über oligotrophe Seen vergeblich nach genauen Angaben, z. B. über Phosphate, suchen. Der Grund dafür ist einfach. Wer sich eingehend mit der Analytik des Phosphats in sehr niederen Konzentrationen befasst, wird rasch auf Schwierigkeiten stossen, die weniger im Prinzip der Methode liegen als in der labormässigen Ausführung. Die Analyse liefert zwar Resultate, welche durchaus glaubwürdig aussehen können; eine Nachprüfung oder Doppeluntersuchung wird indessen ergeben, dass die Resultate fehlerhaft sind. Fehlt zudem die nötige optische Ausrüstung, so ist es nach unseren Erfahrungen fast ausgeschlossen, im Bereich von o-ro oder $20 \mathrm{\gamma} / 1 \mathrm{PO}_{4}-\mathrm{P} \mathrm{zu}$ sichern Werten zu gelangen. Dies ist ohne Zweifel ein Grund dafür, dass wir über die Phosphatverhältnisse ausgerechnet jener Seen, welche heute als Trinkwasserspeicher der Zukunft eine bedeutsame Rolle zu spielen beginnen oder schon spielen, schlecht unterrichtet sind.

In den vergangenen zwei Jahren haben wir uns mit der Methodik der Bestimmung niederster Phosphorgehalte in Seewasser eingehend befasst und sind heute, soweit uns die bisherigen Ergebnisse erkennen lassen, in der Lage, anorganisches Phosphat bis hinunter auf 0,0 mit einer Genauigkeit von mindestens $0,5 \gamma / 1 \mathrm{PO}_{4}-\mathrm{P}$ sicher zu bestimmen, und Gesamtphosphor im nassen Aufschluss mit einer Genauigkeit von rund I $\gamma / 1$ P. Nach diesen 
Erfahrungen ist es somit möglich, $0,5 \gamma / 1 \mathrm{PO}_{4}-\mathrm{P}$ sicher von $0 \gamma / \mathrm{lzu}$ unterscheiden, bzw. I $\gamma / 1$ Gesamt-P von $0 \gamma /$ l. Es handelt sich um die altbekannte Molybdatmethode mit Reduktion der Phosphormolybdänsäure durch Zinnchlorid; die Verfeinerungen, welche die Methode erfahren hat, liegen in der labormässigen Ausführung und in der Messtechnik.

\subsection{Die Rolle des Phosphors als Nährstoff im See}

Ohne dass wir im einzelnen auf die reiche Literatur über dieses Thema hinzuweisen brauchen, ist man sich weitgehend darüber einig, dass von den beiden wichtigsten Nährstoffen im See, Stickstoff. und Phosphor, die Hauptrolle dem letzteren zufällt, erstens, weil er in den meisten Fällen wirklich derjenige Nährstoff ist, der während der Stagnationszeit zuerst aufgebracht wird und von diesem Zeitpunkt an die weitere Produktion von pflanzlichen Zellen und damit von organischer Substanz verlangsamt, zweitens, weil Stickstoff dem See in wesentlichem Ausmass aus dem Kulturboden zufliesst, jedenfalls noch wesentlich mehr als Phosphor, drittens, weil gewisse Algenarten, namentlich aus der Gattung Anabaena, offenbar in der Lage sind, Luftstickstoff zu binden [I5] und auch das Regenwasser Stickstoffverbindungen in beträchtlicher Konzentration enthalten kann, und viertens, weil sich die technische Entfernung des Phosphors heute besser beherrschen lässt als jene des Stickstoffs. Zwar sind die Akten über die Bedeutung des Stickstoffs in Gewässern nicht etwa geschlossen, doch beschränken wir uns aus den erwähnten Gründen in den folgenden Ausführungen auf den Phosphor.

\subsection{Der Phosphor im näbrstoffreicben See}

Ein sehr nährstoffarmer See enthält: entsprechend wenig Organismen pro Volumeneinheit, $d$. h. seine spezifische Produktion ist niedrig, sein Wasser ist klar, und die Organismen verteilen sich auf eine Schicht, die in die Tiefe gemessen 20 und mehr Meter umfassen kann. In einem etwas nährstoffreicheren See vom mesotrophen Typus, in welchem also während der Sommerstagnation sowohl Phosphat als auch Nitrat aufgebraucht werden und der Phosphor grossenteils in organischer Bindung vorliegt [3I], drängt sich die Algenproduktion auf eine weniger mächtige Schicht zusammen und konzentriert sich, wenn Oscillatoria rubescens invadiert, auf eine schmale Zone zwischen Io und Is $\mathrm{m}$. An der Biomasse gemessen steigt die Produktion dann kräftig an. Nehmen die Nährstoffgehalte weiter zu, so ist damit nicht in erster Linie ein weiteres Ansteigen der Or- 
ganismenproduktion irn gesamten verbunden, sondern eine weitere Konzentration der trophogenen Schicht auf die obersten Meter unter der Oberfläche, während infolge der zunehmenden Trübung die unteren Schichten verdunkelt sind und als Lebensraum für pflanzliche Organismen ausfallen. Dieses biologische Verhalten hat seinerseits zur Folge, dass die vorhandenen Nährstoffe während eines grossen Teils der Vegetationszeit nur in einer wenig mächtigen Schicht ïberhaupt ausgenützt werden, wie beispielsweise Phosphatmessungen aus dem Greifensee während und am Ende der Stagnation und während der Vollzirkulation zeigen (Abb. I).

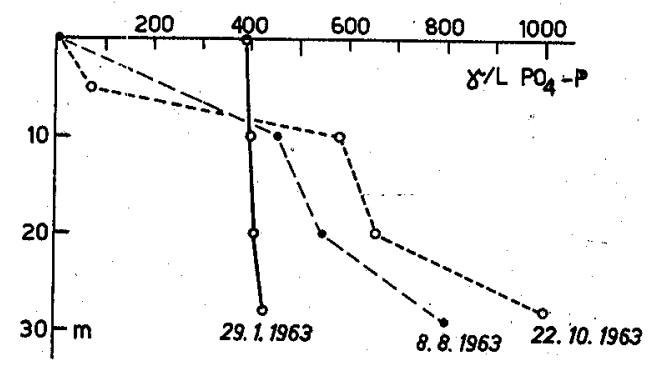

Abbildung 1

Phosphatgehalt im Greifensee während der Zirkulation (29. 1. 63; ausgeglichener Gehalt), wäh . rend der Stagnation (8.8.63) und am Ende der Stagnation (22 10.63).

Für den See, der nachher seine eigenen Produkte wieder abzubauen hat, ist dies zwar gesamthaft gesehen ein Vorteil; indessen sieht ein solches Gewässer während der sommerlichen Planktonentwicklung unansehnlich genug aus, und die Regel ist berechtigt, dass die Produktionsdichte in der obersten Wasserschicht um so höher ist, je höher der Gehalt an Nährstoffen ist. Die vorhandenen Phosphate werden im Lauf von Wochen oder wenigen Monaten aufgezehrt; trotzdem nimmt die Algentrübe des Wassers nicht $a b$. Hier nun begegnen wir einer Erscheinung, die allgemein besonderes Interesse verdient und namentlich in nährstoffarmen Seen zweifellos eine grosse Bedeutung besitzt: Es ist der «kurzgeschlossene» oder «kleine» oder «intrabiozönotische» Kreislauf (z. B. [ro]). Die jetzt, nachdem der ursprüngliche Vorrat aufgezehrt ist, wirksamen Phosphate haben verschiedene Herkunft: einzelne Algen, darunter besonders Oscillatoria, besitzen die Fähigkeit, Phosphat zu speichern, und zwar in derartigen Mengen, dass sie monatelang ohne jegliche weitere Zufuhr gedeihen und sich weiter vermehren können. Die abgestorbenen Organismen sinken nicht erst in die Tiefe $a b$, sondern beginnen sich sofort zu zersetzen. Namentlich 
im Sommer geht diese Zersetzung, wie BACHOFEN [4] an Oscillatoria rubescens nachgewiesen hat, und wie unsere eigenen Beobachtungen bestätigen, sehr rasch vor sich, so dass zumindest ein Teil des Phosphors in gelöster, organischer Form noch innerhalb der trophogenen Schicht wieder frei wird. Untersuchungen von GOLTERMANN an Scenedesmus quadricauda zufolge [I2] werden innerhalb weniger Tage $70-80 \%$ des Phosphors durch Autolyse freigesetzt. Durch Tätigkeit von Bakterien wird aus den gelösten organischen Phosphorverbindungen in rascher Folge anorganisches Phosphat freigesetzt, wie Untersuchungen mit radioaktiv markiertem Phosphat ergeben haben [38]. Die nackten oder dünnhäutigen Nannoplanktonformen, welche in eutrophen Seen im Frühjahr geradezu explosive Massenentfaltungen hervorbringen können und auch sonst in der Produktivität der Seen einen lange unterschätzten, wichtigen Platz einnehmen [2I], sowie die Bakterien dürften sich unmittelbar nach dem Tod zersetzen, womit ihre körpereigenen Nährstoffe sofort wieder zur Verfügung stehen. Untersuchungen von RIGLER [23] zufolge kann Phosphat in eutrophen Gewässern in kürzester Zeit organismisch festgelegt und wieder freigesetzt werden, mehrmals in einer Stunde, wobei neben den Algen auch Bakterien wesentlich beteiligt sind. In nährstoff- und organismenarmen Gewässern kann die Umsetzungszeit bis einige Tage dauern, was immer noch erstaunlich niedrig ist. Als weitere Phosphorquelle kommen die Zooplankter in Frage, welche allnächtlich in die oberen Wasserschichten aufsteigen und nach Messungen von POMERoy, MatHews und MiN [22] täglich ungefähr so viel Phosphor, davon zur Hälfte anorganisches Phosphat, abgeben; wie ihr Körper enthält. Dieser kurzgeschlossene Kreislauf liefert nach den Erfahrungen mancher Autoren und nach unseren eigenen Beobachtungen genügend Phosphate, dass sogar Massenentwicklungen von Algen möglich sind. Dies war in den letzten Jahren in mehreren schweizerischen Seen der Fall. Als letzte, je nach Fall vielleicht wichtigste Quelle von Nährstoffen darf die ständige Zufuhr von aussen her, aus Dränagen, Bächen, Flüssen und Abwassereinläufen nicht unerwähnt bleiben.

\subsection{Der Pbosphor im näbrstoffarmen See}

Wenn auch die Probleme des Nährstoff kreislaufes und der Produktion in überdüngten Seen wissenschaftlich überaus interessant sind und vom Standpunkt der Gewässerreinhaltung aus höchste Aktualität besitzen, so muss man sich doch im klaren sein, dass jede Reinhaltungsmassnahme an einem seit längerer Zeit eutrophen See nicht zu einer plötzlichen 
Wende in seinem Stoff haushalt führen kann, sondern dass «der Weg zuruick» eine geraume Zeit beanspruchen wird:

"Langsamer wird sich das chemisch-biologische Gleichgewicht in den Seen wieder einstellen, weil ja an ihrem Grunde eine unterschiedlich mächtige Schicht von Faulschlamm abgebaut; das heisst mineralisiert werden muss. Aber auch in den Seen werden sich mit der Zeit die Verhältnisse wieder bessern, und zwar um so rascher, je schneller infolge bedeutender, reiner Zuflüsse das Wasser sich in ihnen erneuert» (O. JAAG [I6]).

Um die Verhältnisse in der Schweiz in den Vordergrund zu stellen: bei jenen Seen, welche als ausgesprochen eutroph bekannt sind, handelt es sich in der Mehrzahl um ziemlich flache Gewässer, welche schon natürlicherweise zum eutrophen Typus neigen [Io], und welche bis jetzt nicht in grösserem Masse zur Trinkwasserversorgung herangezogen wurden. Neben diesen Gewässern, welche man als die «stillen Reserven der Zukunft» betrachten darf, besitzt unser Land nun aber grössere, tiefere Seen, deren Stoff haushalt zum Teil zwar auch schon etwas stärker belastet ist, und die allerhand Zeichen dafür tun, dass auch sie sich in einer ungünstigen Entwicklung befinden' (z. B. [2]), die sich aber zur Gewinnung von Trinkwasser heute noch hervorragend eignen und deshalb besonders schutzwürdig sind. Es sind dies der Bodensee, der Vierwaldstättersee, der Thunersee, der Walensee, der Neuenburgersee und der Genfersee.

Uber den Phosphathaushalt des Bodensees z. B. hat die «Internationale Gewässerschutzkommission für den Bodensee» während zweier Jahre eingehende Untersuchungen durchführen lassen; ihre Resultate befinden sich gegenwärtig in Ausarbeitung. In ihrem Bericht Nr. I [2] hält die Kommission fest, dass der mittlere Gehalt an anorganischem Phosphat 1959 bei $9 \gamma / 1 \mathrm{lag}$, während frühere Bestimmungen wesentlich niedrigere Werte geliefert hatten. Der Phosphatgehalt des Bodensees ist - auch bei vorsichtiger Interpretation der Werte - im Zunehmen begriffen.

Diese "vorsichtige Interpretation» drängt sich deshalb auf, weil die Bestimmungsmethodik auch im Bereich der erwähnten $9 \gamma / \mathrm{l}$ wie schon erläutert ziemliche Unsicherheiten birgt. Unsere eigenen, im Rahmen der Untersuchung der Bodenseekommission durchgeführten Erhebungen ergaben, um dies zu illustrieren, die in Tab. I auszugsweise wiedergegebenen Zahlen.

Aus dieser Zusammenstellung ist ersichtlich, dass die Streuungen, welche die in guten Treuen erarbeiteten Phosphatwerte früher auszuführen schienen, methodisch bedingt waren. Anfänglich lieferte offensichtlich auch die verfeinerte Methodik noch unrichtige Werte, und erst im Lauf der 
Tabelle 1

Bodensee-Obersee, Seemitte zwiscben Langenargen und Arbon; Pbospbat-P $(\gamma / l P)$

\begin{tabular}{|c|c|c|c|c|c|c|c|c|}
\hline $\begin{array}{l}\text { Wasser- } \\
\text { tiefe }\end{array}$ & $\begin{array}{l}1962 \\
21.5 .\end{array}$ & 19.6. & 19.7 & $\begin{array}{l}1963 \\
\left.21.8 .^{*}\right)\end{array}$ & 26,9 & 24. 10 . & 26.11. & $\begin{array}{l}1964 \\
23.4 .\end{array}$ \\
\hline $0 \mathrm{~m}$ & 0 & 5 & 0 & 0,0 & 0,0 & 2,5 & 12,0 & $1.5,0$ \\
\hline $100 \mathrm{~m}$ & 10 & Spur & 2 & 7,5 & 8,5 & 15,0 & 16,5 & 19,5 \\
\hline $150 \mathrm{~m}$ & 10 & Spur. & Spur & 7,5 & 12,0 & & 15,0 & 22,5 \\
\hline $200 \mathrm{~m}$ & 15 & 5 & 0 & 12,5 & 13,5 & 22,0 & 26,5 & 27,5 \\
\hline \multicolumn{9}{|c|}{ erbesserung der Feinmethode } \\
\hline
\end{tabular}

Monate gelang es, mit zunehmender Erfahrung zu einigermassen stabilen Zahlen zu kommen. Jedenfalls sind die Phosphatgehalte des tieferen Hypolimnions, hier also bei Ioo und $150 \mathrm{~m}$, normalerweise ziemlich konstant; die starke «Zunahme» im Lauf der Zeit kann deshalb nicht reell sein, sondern geht auf die Methodik zurück. Die Phosphatwerte liegen denjenigen des Vierwaldstättersees sehr nahe, weshalb ich mich in den folgenden Ausführungen auf diesen See, den wir chemisch besser kennen, beziehen möchte.

Wir untersuchen den Vierwaldstättersee seit $196 \mathrm{r}$ monatlich, und zwar an der tiefsten Stelle im sog. "Kreuztrichter», dem kreuzförmigen Seebecken vor Luzern. Die anorganischen Phosphate werden seit I963 mit der Feinmethode bestimmt, der Gesamtphosphor im Rohwasser und im Membranfiltrat (ausgewaschene Millipore-Membranfilter) seit Juli I963. Bei diesen Untersuchungen hat sich gezeigt, dass die Phosphorkomponenten mit Ausnahme vielleicht der Werte ab so $\mathrm{m}$ Tiefe, welche im Lauf der Zeit ziemlich konstant bleiben, unerwartet starken Schwankungen unterliegen.

In Abb. 2 sind die entsprechenden Phosphatgehalte graphisch dargestellt. Diese Kurven sagen, für sich allein betrachtet, allerdings nicht viel aus, denn der anorganische Phosphatphosphor ist ja nur eine einzige Komponente in einem sehr dynamischen Mehrkomponentensystem, das noch Phosphor in organischer, und zwar in gelöster und ungelöster Form umfasst. Die zeitlich entsprechenden Gehalte an organisch festgelegtem, ungelöstem Phosphor sind in Abb. 3 in gleicher Weise dargestellt; zudem sind in einzelnen dieser Diagramme die Biomasse-Summenwerte (Biomasse des Phytoplanktons, aus den Individuenzahlen berechnet) angegeben. Diese ergeben eine gute Úbereinstimmung mit den Phosphorzahlen und bestätigen damit deren Richtigkeit.

Ohne auf biologisch oder limnologisch interessante Einzelheiten näher einzutreten, haben diese Kurven eines gemeinsam, nämlich eine starke Verschiebung von Monat zu Monat. Während sämtliche übrigen chemischen Komponenten im Seewasser von Monat.zu Monat zwar ebenfalls 


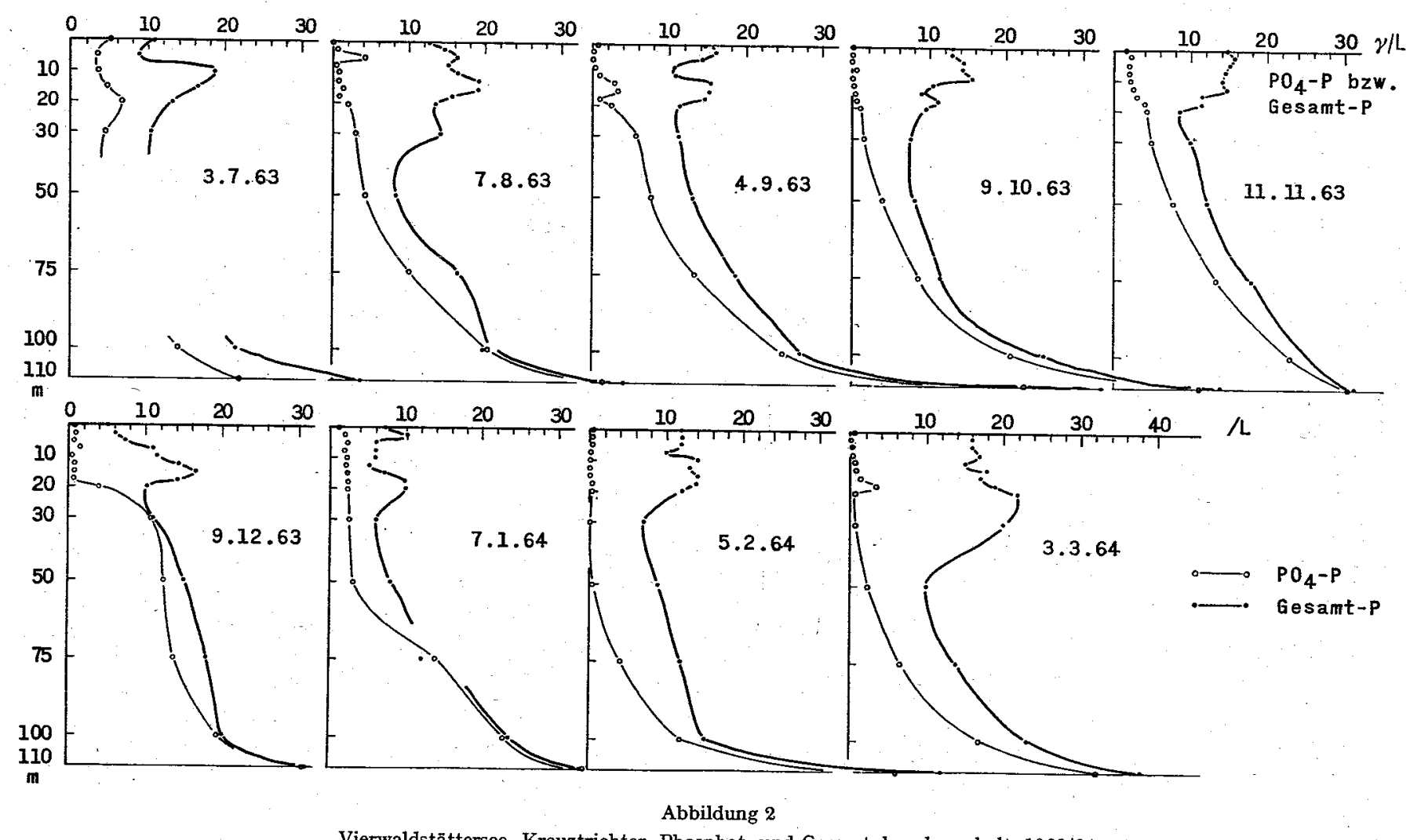

Vierwalđstättersee, Kreuztrichter. Phosphat- und Gesamtphosphorgehalt, 1963/64. 


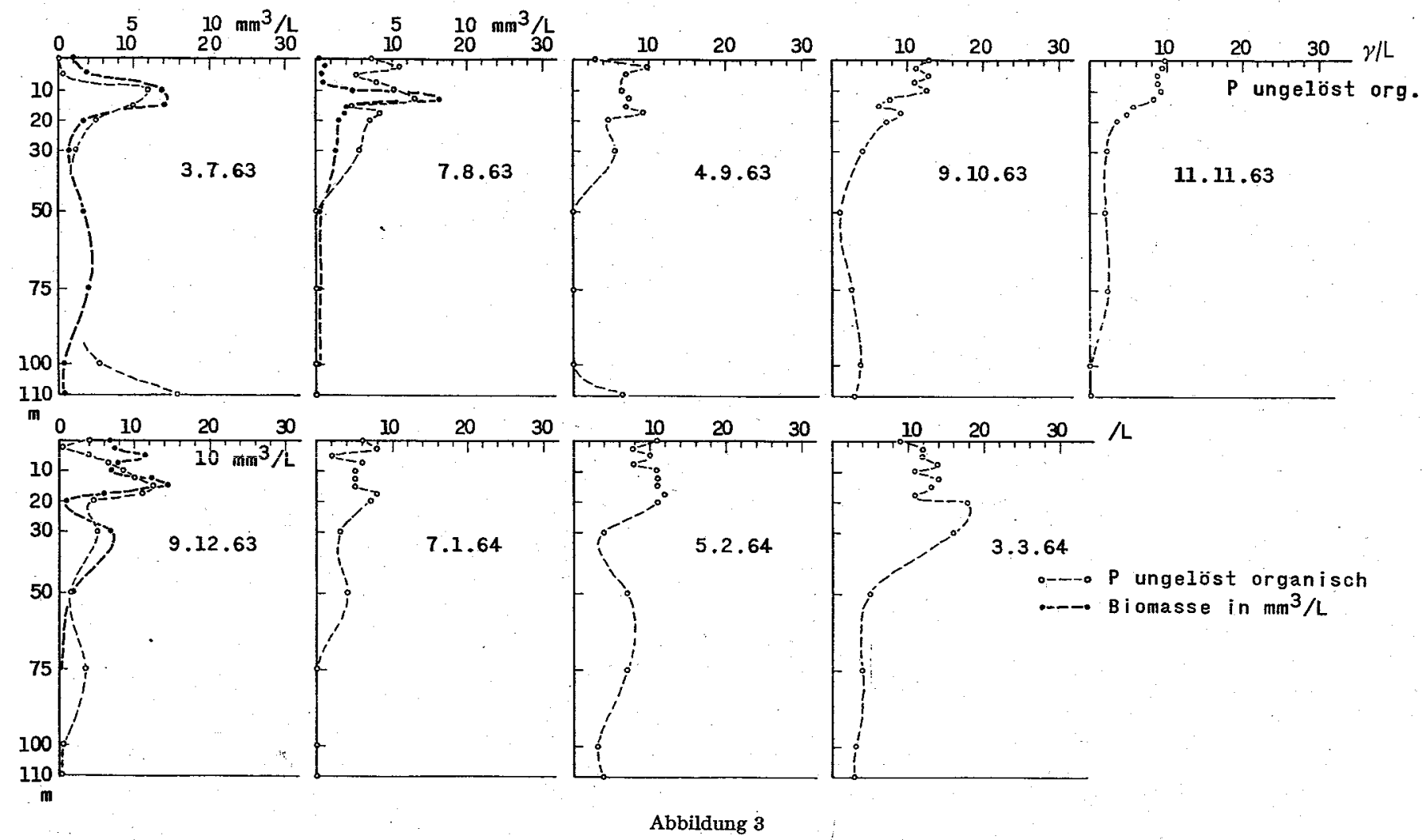

Vierwaldstăttersee, Kreuztrichter. Gehalt des Seewassers an organisch gebundenem, ungelöstem Phosphor und an Biomasse. 
Veränderungen zeigen, die aber langsam und stetig, keinesfalls sprunghaft verlaufen, ist hier genau das Gegenteil der Fall. Zieht man allerdings in Betracht, dass auch in nährstoffarmen Seen rasche Änderungen in der quantitativen Zusammensetzung des Planktons möglich sind, so erstaunt dieser Tatbestand schon weniger, zumal Phosphor, wie wir bereits ausführten, am Stoffgeschehen des Sees ganz besonders intensiv beteiligt ist.

Aus Abb. 2 und 3 geht weiter hervor, dass in der Tiefe des Sees Phosphor angereichert wird, und zwar liegt er hier, als Produkt des aeroben Abbaues, fast vollständig als anorganisches Phosphat vor. Dieses « Depot» nimmt im Laufe der Zeit nicht wesentlich zu; dem steht aber gegenüber, dass der Gesamtphosphor in der trophogenen Schicht (etwa O-20 m Wassertiefe) im Gesamten nicht abnimmt. Praktisch der ganze freie und gebundene Phosphor in dieser Schicht ist dauernd im Unsatz, umd zwar innerhalb des kurzgeschlossenen, intrabiozönotischen Kreislaufes. Die wenigen $\gamma / 1$ ( $1=$ I milliardstel Gramm $=$ I tausendstel Milligramm) genügen, um die Produktion des Sees dauernd aufrechtzuerhalten. Dabei ist diese Produktion, wie Messungen von I. FINDENEGG (mündliche Mitteilung) ergeben haben und wie auch unsere Biomassewerte in Abb. 3 zeigen, gar nicht gering. Die Biomasse in der trophogenen Schicht liegt in der gleichen Grössenordnung, meistens sogar fast gleich hoch wie die - allerdings aus andern Spezies gebildete - Biomasse z. B. des Pfäffikersees oder des Zürichsees. Bei der Interpretation der erwähnten Schwankungen in Abb. 2 und 3 ist nun allerdings zu berücksichtigen, dass die Wassersäule an der Probestelle, die jeweils mit dem Boot genau eingepeilt wird, durch Winde, durch unterseeische Strömungen und durch Pendelschwingungen des Wasserkörpers verschoben wird und die Proben deshalb von Untersuchung zu Untersuchung aus einer etwas andern Wassermasse stammen. Eine Untersuchungsreihe, welche wir innerhalb von drei Tagen an fünf je einen Kilometer weit auseinanderliegenden Punkten ausführten(Situation s. Abb. 4a) hat ergeben, dass von Stelle zu Stelle der Phosphatgehalt auf der gleichen Wassertiefe bis zu $3 \gamma / 1$ (bis $20 \mathrm{~m}$ Wassertiefe), bzw. bis zu Io $\gamma / 1$ (IOO m) und der Gehalt an organischem Phosphor bis zu $6 \gamma / 1$ (bis $20 \mathrm{~m}$ Wassertiefe), bzw. bis zu $2 \gamma / 1$. (IOO m) schwanken kann.

Immerhin finden sich, wie die in Abb. $4 b$ dargestellten Resultate zeigen, keine Parallelverschiebungen der ganzen Verlaufskurve, so dass Schwankungen, wie sie in den Abbildungen 2 und 3 von Monat zu Monat auftreten, wirklich auf biologisch bedingte Veränderungen zurückgehen.

$\mathrm{Zu}$ diesen Zahlen liesse sich einwenden, dass es sich hier bloss um sehr : geringe Konzentrationen handelt. Es darf aber wiederum nicht übersehen 


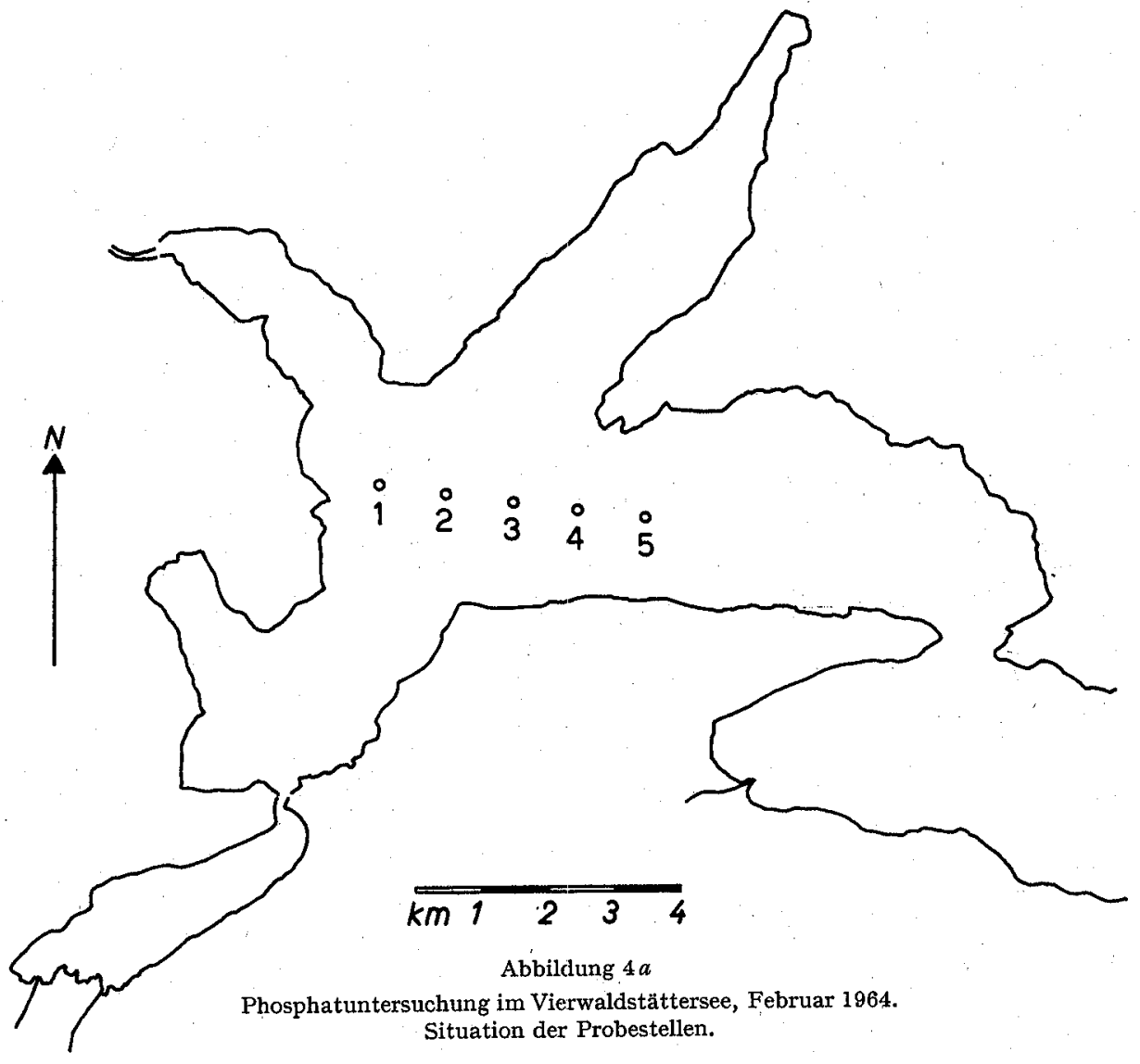

werden, dass diese winzigen Mengen Phosphor offensichtlich genügen, um das Wachstum des Phytoplanktons, das uns heute wegen des überaus starken Auftretens der Burgunderblutalge bereits erhebliche Sorgen macht, in Gang zu halten. Ob freilich allein der Phosphor für diese Produktion verantwortlich ist, wissen wir nicht. Es wäre denkbar, dass auch andere Stoffe, namentlich Spurenelemente, für Vegetieren oder Gedeihen des pflanzlichen Lebens und damit der ganzen Biozönose im See mitverantwortlich sind, bzw. dass noch andere Stoffe die Produktion beeinflussen können. So kann . Eisen, welches beispielsweise im Vierwaldstättersee nur in Spuren vorhanden ist, nach Untersuchung von SCHELSkE, HOOPER und HaERTL [24] die Produktion eines ganzen Sees erhöhen, wenn es als Chelat zugegeben wird. Auch ELSTER [Io] weist auf die Möglichkeit hin, dass Eisen als pro- 

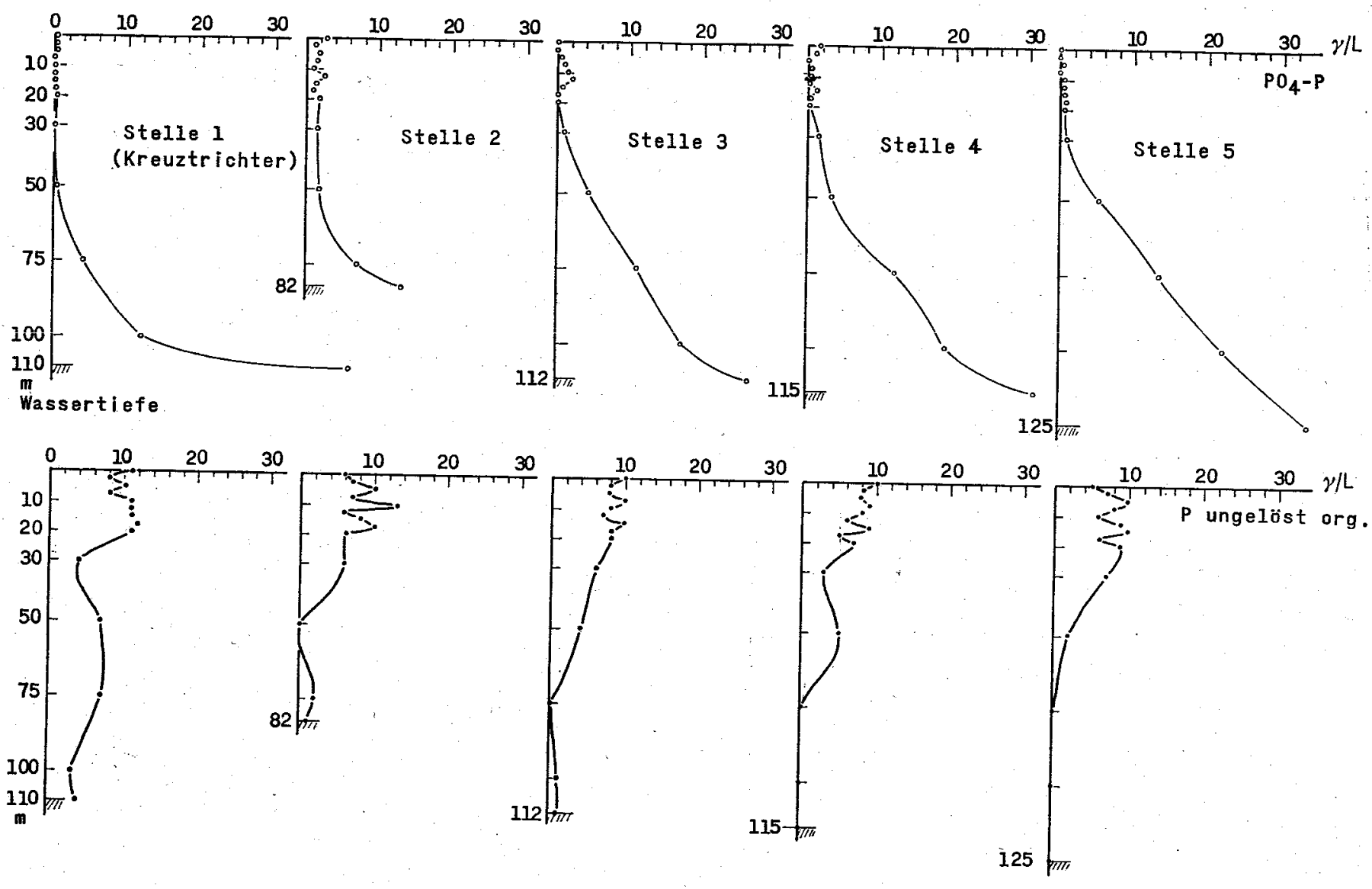

Abbildung $4 b$

Vierwaldstättersee, Februar 1964. Gehalt an Phosphat- und an ungelöstem organischem Phosphor an 5 benachbarten Punkten. 
duktionsbegrenzender Faktor wirken kann, ein Problem, das gerade im $\mathrm{Zu}$ sammenhang mit der Phosphorelimination durch Eisenflockung ein weiteres Studium erfordert. Als Bestandteil des lebensnotwendigen Vitamins $B_{12}$ scheint im limnischen Stoffkreislauf das Kobalt manche Fragen aufzugeben, zumal dieses Metall, als Cobalamin im Plankton, bzw. im Wasser vorhanden, im Lauf des Jahres ähnliche Konzentrationsschwankungen ausführt wie das Phosphat [36]. In den Gewässern liegt der Gehalt an Vitamin $\mathrm{B}_{\mathbf{1 2}}$ wesentlich niedriger als der für Algenkulturen optimale Gehalt [6], und zudem ist ein beträchtlicher Teil des gesamten im Wasser vorhandenen Kobalts in den limnischen Stoffumsatz eingespannt [r3]. Die Vermutung ist deshalb nicht ganz abwegig, dass auch Kobalt oder das Vitamin $\mathrm{B}_{12}$, möglicherweise auch noch andere Stoffe, die Produktion begrenzen können.

Die vorstehend geschilderten Phosphorverhältnisse dürfen, soweit wir aus den vorhandenen Daten entnehmen können, ohne wesentliche Einschränkung auch auf den Bodensee übertragen werden. Aüch dort scheint der «kurzgeschlossene Kreislauf» eine bedeutsame Rolle zu spielen, und ähnliches gilt sinngemäss für alle übrigen zumeist meso- oder eutrophen Seen, mit der Einschränkung allerdings, dass hier der kurzgeschlossene Kreislauf zwar auch spielt, aber solange von untergeordneter Bedeutung ist, als noch genügend Nährstoffe im Überschuss vorhanden sind. Wenn dann aber dieser von der Vollzirkulation her bestehende Vorrat aufgebraucht ist, tritt der kleine Kreislauf auch hier in seiner vollen Bedeutung zutage: die Vorstellung nämlich, dass mitdem Verbrauch des Phosphors die Produktion auf hört, würde zwar theoretisch dem Gesetz des Minimums entsprechen; sie ist aber unrichtig, indem im kurzgeschlossenen System dauernd so viel Phosphor bereitgestellt wird, dass eine ansehnliche Produktion bestehen kann.

Natürlich weiss man schon lange urn die Existenz des kleinen Kreislaufs; unsere Feststellung ist beileibe nicht neu. Neu daran ist höchstens die sichere Kenntnis der Phosphatverhältnisse. Die praktischen Folgerungen, die sich daraus ergeben, werden im nächsten Kapitel erörtert.

\section{Die praktischen Folgerungen}

Im letzten Abschnitt haben wir uns eingehend mit den Nährstoffverhältnissen im Vierwaldstättersee befasst und diese sogar auf den Bodensee (Obersee) übertragen. Die kleineren eutrophen Seen wurden dabei eher am Rande behandelt. Dies hat seine besonderen Gründe: erstens sind uns manche dieser Seen aus früheren Arbeiten bereits bekannt und zweitens 
wollten wir uns bewusst mit jenen Seen befassen, welche gegenwärtig zur Trinkwassernutzung in grossem Umfang herangezogen werden oder dafür in Frage stehen, und in denen die Überdüngung, bzw. Eutrophie noch nicht so weit vorangeschritten ist, als dass bereits Perioden anaerober Verhältnisse in der Seetiefe auftreten würden, somit Seen, welche - grob gesagt noch keine Therapie benötigen, sondern denen mit prophylaktischen Massnahmen schon viel geholfen wäre.

Aus bereits dargelegten Gründen beschränken wir uns im folgenden wiederum auf den Phosphor.

\section{I Die Notwendigkeit der Fernhaltung der Nährstoffe vom See}

Uber die Notwendigkeit, den Seen nicht nur die Schmutz-; sondern auch die Nährstoffe fernzuhalten, kann heute nicht mehr diskutiert werden: Geht man nämlich vom Grundsatz aus, dass die Behandlung der Abwässer den Besonderheiten des Vorfluters Rechnung zu tragen hat, so besteht nicht mehr der geringste Zweifel, dass die Seen nicht nur vor den Schmutzstoffen aus dem Abwasser, sondern genau so gut auch vor den Pflanzennährstoffen zu schützen sind, vorerst ungeachtet der technischen und finanziellen Konsequenzen, welche daraus erwachsen. In die Zukunft gesehen spielt die Reinhaltung der Seen eine gleichbedeutende Rolle wie die Reinhaltung des Grundwassers. Nachdem man unter dem Druck der Ereignisse an den verantwortlichen Stellen den Ernst der heutigen Lage auf dem Grundwassersektor eingesehen hat, man denke nur an die sich hartnäckig mehrenden Ölunfälle, wagen wir zu hoffen, dass die gleiche Erkenntnis auf dem Gebiet der Reinhaltung der Seen, unserer wichtigsten Trinkwasserreserven, Wurzeln schlagen wird.

Aber wenn auch alle möglichen Reinhaltungsmassnahmen realisiert würden, so wären damit die Probleme der Eutrophie der Seen noch nicht gelöst - die Nährstoffe fernzuhalten ist nur ein Teil des Ganzen. Zur limnologischen Beurteilung der Nährstoffelimination gehören weitere Aspekte, welche sich von der eigentlichen Elimination, bzw. Fernhaltung nicht trennen lassen.

\subsection{Die Herkunft der Nährstoffe}

Als Quellen namentlich von Phosphor kommen in Frage

a) direkt einfliessende $A$ bwässer aus Siedlungen und Industrie in der Uferzone,

b) Zuflüsse aus dem weiteren Einzugsgebiet, welche ihrerseits durch Abwässer belastet sind,

c) Dränagewasser und Abschwemmung aus dem Kulturboden. 
Wie stark diese einzelnen Quellen an der Zufuhr zu einem See beteiligt sind, hängt von den Verhältnissen im Einzugsgebiet ab. Von den einzelnen Fraktionen nun ist ein Teil mit technischen Mitteln erfassbar: alles, was mit Kanalisationen gesammelt werden kann, kann gereinigt oder dem See mit einer Ringleitung überhaupt ferngehalten werden. Der See ist aber nicht nur das Auffangbecken für die Uferregion, sondern für sein ganzes, mitunter sehr grosses Einzugsgebiet. Schwieriger wird es deshalb bei $\mathrm{Zu}$ flüssen, welche ein grosses Gebiet entwässern: während in unmittelbarer Nähe eines Sees, d. h. in den Ufergemeinden, die Notwendigkeit einer Nährstoffelimination oder Fernhaltung erfreulicherweise immer mehr eingesehen wird, kann diese Einsicht fernab vom See noch nicht im gleichen Mass erwartet werden. Die grösseren Zuflüsse bilden deshalb, auch wenn die in ihnen mitgeführten, gereinigten oder ungereinigten Abwässer zur Nährstoffelimination theoretisch erfassbar wären, vorderhand noch eine wichtige Nährstoffquelle.

Der dritte Anteil der Nährstoffe stammt aus dem Kulturboden, aus der landwirtschaftlichen Düngung, aus abgeschwemmter Erde usw. Die Nährstoffe gelangen hier nicht in eine Kanalisation, sondern in Bäche und Flüsse und kommen völlig unkontrollierbar in den See.

Untersuchungen von AMBÜHL [3] am Hallwilersee ergaben, dass insgesamt $45 \%$ der Phosphorzufuhr (I956/57) - jene aus dem Baldeggersee, der in den Hallwilersee entwässert, nicht eingerechnet - durch Bäche aus dem Gelände in den See kommen und technisch nicht zu erfassen sind. Ähnliche Erhebungen der EAWAG an den Zuflüssen zum Murtensee zeigten, dass mit einer konsequent durchgeführten mechanisch-biologischen Reinigung sämtlicher Abwässer des ganzen Einzugsgebietes rund $25 \%$ der totalen Phosphorzufuhr überhaupt erfassbar wären; mit einer ebenso konsequenten zusätzlichen Phosphorelimination (Wirkungsgrad 90\%) könnten rund $47 \%$ der Phosphorverbindungen zurückgehalten werden. Der Rest gelangt auf jeden Fall in den See. Allerdings sind die Voraussetzungen im Einzugsgebiet des Murtensees besonders ungünstig.

Zumindest ein Teil der Nährstoffe, die auf diese Weise den See belasten, stammt aus der landwirtschaftlichen Düngung. So stellt CoughLIN [7] fest, dass der Phosphatgehalt in untersuchten Oberflächengewässern etwa zur Hälfte auf die Abschwemmung aùs ländlichen Gebieten zurückzuführen ist. Die«Ontario Water Resources Commission» führt die zunehmende Veralgung der grossen Seen Nordamerikas unter anderem auf den Gebrauch von Kunstdüner in den Landwirtschaftsgebieten zurück [I], und HUBER [I4] findet, dass die Schwemm- und Dränagewässer aus landwirtschaftlich 
genutzten Flächen bis zu 45\% des Gesamt-P-Gehaltes der Oberflächengewässer verursachen. Nach den Untersuchungen von Voss [37] gehen etwa $2 \mathrm{I} \%$ des von der Schussen in den Bodensee transportierten Gesamtphosphors zu Lasten der Abschwemmung aus landwirtschaftlich genutzten Gebieten (I958/62).

Diese wenigen Zahlen, die uns wenigstens die zu erwartende Grössenordnung angeben, lassen erkennen, dass es nicht gleichgültig ist, ob ein See von seiner direkten Abwasserlast befreit wird oder nicht, dass aber seine restliche Belastung, welche er von anderen Quellen her erfährt, in der gleichen Grössenordnung liegt und deshalb die gleiche Beachtung verdient.

\subsection{Elimination oder Fernhaltung?}

Befassen wir uns zunächst mit jenem Teil des Phosphors, der mit den heute zur Verfügung stehenden Mitteln erfasst werden kann. Ist eine Ufergemeinde, eine Stadt oder eine ganze Seeregion kanalisationstechnisch zusammengefasst, so stehen grundsätzlich zwei Möglichkeiten offen, den See vor dem Abwasser zu schützen, nämlich

a) Vollreinigung des Abwassers inklusive Elimination des Phosphors und Abfluss in den See, und

b) entweder biologische Reinigung und Weitertransport des gereinigten Abwassers mit einer Ringleitung bis zum Seeausfluss, oder Sammeln des Rohwassers aus der gesamten Seeregion in einer Ringleitung und Behandlung in einer Reinigungsanlage unterhalb des Sees.

Welche dieser Möglichkeiten in Frage kommt, wird wohl von den lokalen Gegebenheiten abhängen. Es darf aber nicht übersehen werden, dass die beiden Verfahren, vom Standpunkt der Reinhaltung des Sees aus gesehen, nicht gleichwertig sind. Zwar wird auch eine Ringleitung, besonders wenn sie mit Hebewerken ausgeruistet werden muss, nicht absolut sicher funktionieren und im Falle einer Pumpenstörung ihr Abwasser für eine gewisse Zeit über die Entlastung direkt in den See entlassen müssen, doch besteht für jene Zeit, in der sie wirklich funktioniert, die beste Gewähr dafür, dass wirklich keine Abwässer, damit auch kein Abwasserphosphor und keine weiteren physiologisch wirksamen Stoffe zum See gelangen, während eine chemische Elimination naturgemäss eher zu Störungen neigt und auch nicht den Höchsteffekt einer Ringleitung erreichen kann. Damit soll kein Werturteil ausgesprochen werden. Es soll an dieser Stelle auch nicht untersucht werden, ob eine Kanalisation nach dem Misch- oder nach 
dem Trennsystem vorteilhafter wäre. Wo jedenfalls auch nur entfernt die Möglichkeit besteht, Abwasser einem See vollständig fernzuhalten, sei es mit einer Ringleitung oder auch, indem man es in ein benachbartes Einzugsgebiet ohne See hinüberleitet, sollte dieser Weg, wie es am Hallwilersee $[5$, II] und am bayrischen Tegernsee [I3] bereits der Fall ist und für den

- Thunersee sowie für die Region Luzern [25] geplant wird [26], unbedingt beschritten werden. Wohingegen das System der Ringleitung nicht möglich ist, und das betrifft das gesamte Hinterland eines Sees, können die Phosphate nur auf chemischem Wege entfernt werden. Hier liegt unseres Erachtens das eigentliche Anwendungsfeld für diese Verfahren.

Die Frage, wie weit die chemische Elimination getrieben werden soll, beantwortet sich leicht: Wir haben gesehen, dass schon geringe Mengen Phosphor im See genügen, um - auf dem Wege über den « kurzgeschlossenen Kreislauf» - die Produktion organischer Substanz immer in Gang zu halten. Wir haben festgestellt, dass derjenige Anteil an Phosphor, der nicht eliminiert werden kann, weil man ihn nicht in die Hand, bzw. in den Sammelkanal bekommt, in der gleichen Grössenordnung liegt wie derjenige aus dem Abwasser. Wir müssen uns ferner darüber im klaren sein, dass der Anteil an Phosphor, der aus Waschmitteln ins Abwasser gelangt, immer weiter ansteigt. Nach Voss beträgt der̀ aus den modernen, synthetischen Waschmitteln stammende Anteil des Phosphors in der Schussen (Zufluss zum Bodensee) ungefähr $35 \%$ und nimmt weiter zu [37], um nur diese eine Zahl zu nennen.

Angesichts dieser Tatsache nun « grosszügig» sein und die Anforderungen an die Eliminationsleistung einer Kläranlage bescheiden halten zu wollen, wäre unseres Erachtens nicht nur falsch, sondern unverantwortlich. Natürlich muss man sich im klaren sein, dass ein Teil der Düngstoffe, welche unsere Seen schädigen, nicht erfasst werden kann. Auf der anderen Seite sind wir aber im Interesse der Volkshygiene darauf angewiesen und auch moralisch dazu verpflichtet, die Seen mit allen Mitteln vor weiterer Schädigung zu bewahren. Das kann aber nicht anders geschehen als durch völliges Ausschöpfen aller jener Mittel, die uns wirklich zur Verfügung stehen. Auf die Phosphorelimination bezogen heisst das aber, dass das verwendete Verfahren auf äusserste Leistung zu trimmen ist, ohne zu sehr auf die wirtschaftliche Seite zu achten. Wirtschaftliche Gesichtspunkte mögen heute noch eine Rolle spielen. Es ist aber nicht zu verkennen, dass überall in unserem Lande der Wasserpreis erhöht wird, und verschiedentlich haben sich schon Fälle ereignet, da man gerne einen hohen Preis bezahlt hätte, wenn man nur wenigstens Wasser gehabt hätte! 


\subsection{Die Frage der Regenauslässe}

Aber auch bei vollständiger Elimination oder Fernhaltung ist der See nicht völlig von den Abwassernährstoffen entlastet: bei Regenwetter springen die Regenentlastungen im Kanalisationsnetz an und entlassen das anfänglich konzentrierte, später verdünnte Abwasser ungereinigt in den See. Der Anteil an der jährlichen Schmutzwassermenge, welcher auf diese Weise nicht in die Kläranlage, sondern ins Gewässer gelangt, beträgt nach Angaben von A. HÖRLER IO-20\%; ist also sehr beträchtlich. Diesen Anteil zu verkleinern, z. B. durch Einschaltung gut dimensionierter Regenwasserrückhaltebecken mit späterer Rückgabe des aufgefangenen Wassers an die Kläranlage, wie z. B. W. SPRING [25] anhand des Projektes für die Abwasserbeseitigung in der Region des unteren Thunersees erläutert, ist ein grosses Anliegen des Limnologen an den Ingenieur.

\subsection{Der nicht erfassbare Anteil}

Besondere Sorgen bereitet uns der schon mehrmals erwähnte Anteil an Nährstoffen, der aus Dränagen, durch Abschwemmung von Kulturland usw., als Fluss, Bach oder Sickerwasser in die Seen gerät. Er entzieht sich unserem Zugriff völlig; er fällt demnach nicht unter das Kapitel der Nährstoffelimination und könnte deshalb hier übergangen werden. Indessen ist dieser Anteil, wie wir bereits ausführten, derart bedeutend, unter Umständen grösser als der klärtechnisch erfassbare, dass sich auch hierüber einige Úberlegungen aufdrängen.

Derjenige Phosphor, der im See nicht im kurzgeschlossenen Kreislauf verbleibt, sondern mit abgestorbenen Organismen in die Tiefe verschoben wird, reichert sich dort an und bildet ein Depot, das frühestens mit der nächsten Vollzirkulation wieder auf die ganze Wassermasse verteilt wird. ZurSanierung von eutrophen Seen, welche diese Erscheinung gemäss Tab. 2 in besonders ausgeprägtem Masse zeigen, wurde schon vor geraumer Zeit von ZIGERLI [40], später von THOMAS [28, 35] vorgeschlagen, mit einer Rohrleitung das nährstoffreiche, sauerstoffarme Tiefenwasser abzuführen.

Tabelle 2

Extreme Phospbatwerte in eutropben Seen

\begin{tabular}{llll}
\hline See & & Oberfläche & Uber Grund \\
\hline Rotsee & Herbst 1963 & Spuren & $2850 \gamma / 1 \mathrm{P}(16 \mathrm{~m}$ tief $)$ \\
Greifensee & Herbst 1963 & $8 \gamma / 1$ & $1005 \gamma / 1(29 \mathrm{~m})$ \\
\hline
\end{tabular}


Dieses Verfahren, dessen technische und wirtschaftliche Aspekte von WLDI [39] untersucht worden sind, ist vermutlich das einzige, das einen eutrophen See in absehbarer Zeit von seiner eigenen Nährstofflast erleichtern kann. Da nun diese Anreicherung von Phosphor, wie Abb. 2 und 3 zeigen, auch in Seen mit geringerem Nährstoffgehalt auftritt, wäre es zumindest zu überlegen, ob der See nicht mit einer Absaugleitung nährstoffmässig entlastet werden könnte. Wir haben hierzu für das Luzerner Becken des Vierwaldstättersees einige Berechnungen angestellt, welche auf den Phosphoranalysen vom 7. Januar 1964 beruhen.

Der tiefste Teil dieses Seebeckens enthält, auf eine Höhe von $18 \mathrm{~m}$, vom tiefsten Punkt aus berechnet, total 27,5 $\cdot 10^{6} \mathrm{~m}^{3}$ Wasser. Aus den einzelnen

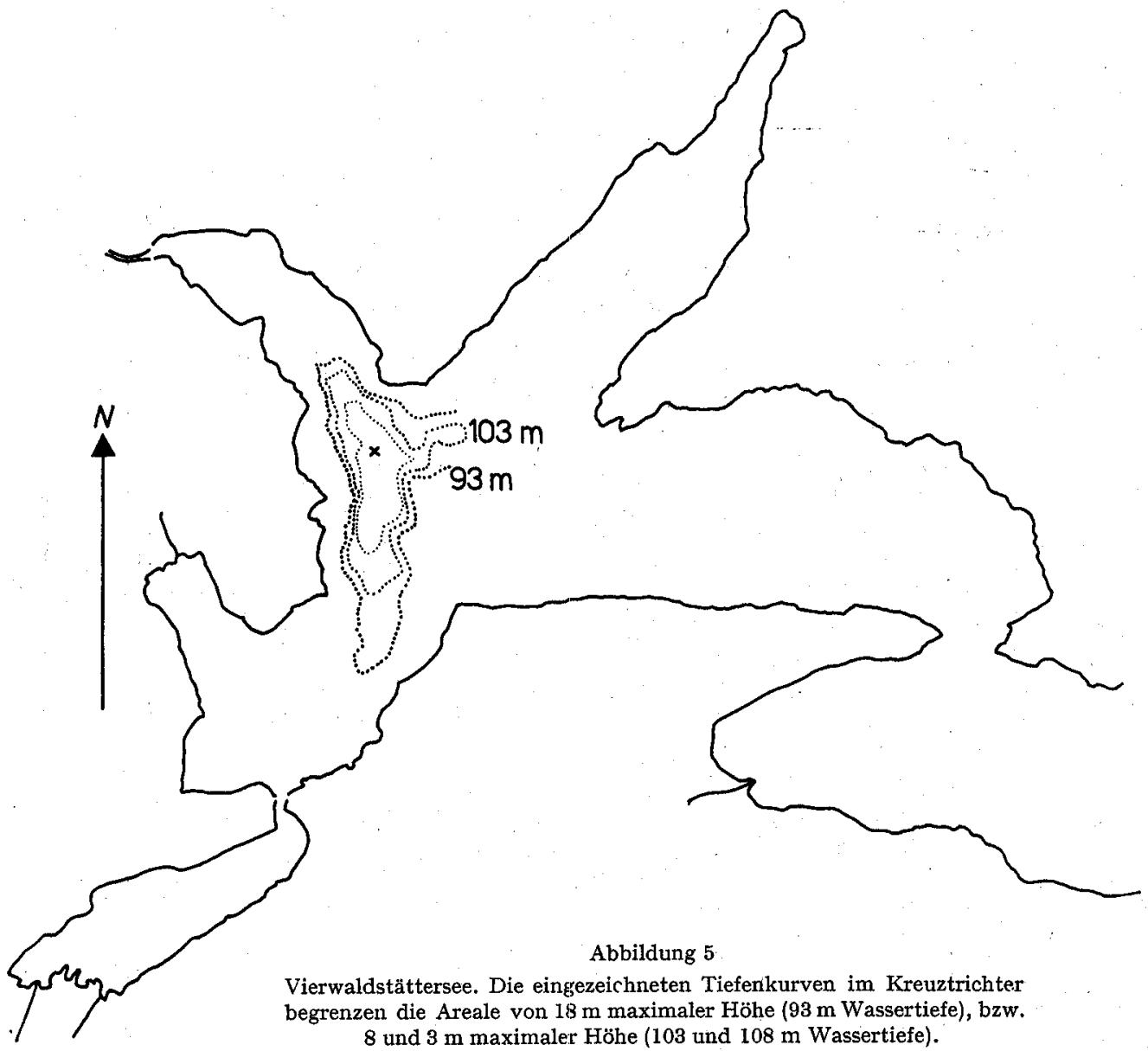


Schichten und den dazugehörigen Gesamt-P-Konzentrationen ergibt sich für die Schicht von III bis $93 \mathrm{~m}$ Wassertiefe ein mittlerer Gehalt von 23, I $\gamma /$ l oder eine Gesamtmenge von total $635 \mathrm{~kg} \mathrm{P.}$

Mit einer Fördermenge von $\mathrm{I} \mathrm{m} \mathrm{m}^{3} / \mathrm{s}$ könnte dieses Wasservolumen in etwa $10^{1} / 2$ Monaten abgezogen werden. Schon für diese relativ geringe Menge ergäbe sich eine beträchtliche, mindestens $4,8 \mathrm{~km}$ lange Leitung. Die Reuss transportiert dagegen bei einer mittleren Wasserführung von III $\mathrm{m}^{3} / \mathrm{s}$ und einem mittleren Gehalt von I3 $\gamma / \mathrm{l}$ Gesamt-P jährlich 45,5 Tonnen $P$ weg. Die mit der Absaugleitung erfasste Menge würde nur I, $5 \%$ von diesem oberflächlichen Wegtransport ausmachen. Die gesamte Zufubr zum See ist in jedem Fall, wie Berechnungen in kleineren Seen und am Bodensee [I7] gezeigt haben, wesentlich grösser als der oberflächliche Wegtransport; somit würde der Nutzeffekt noch geringer ausfallen. Eine Erhöhung der hydraulischen Absaugleistung um ein $n$-faches hätte nicht einen $n$-fachen Wegtransport von Phosphor zur Folge, da entsprechend mehr höhergelegenes, phosphorarmes Wasser erfasst würde. Auch wenn man berücksichtigt, dass sich der Vierwaldstättersee infolge seiner starken ober- und unterseeischen Gliederung für solche Überlegungen schlecht eignet, lässt sich doch erkennen, dass eine Tiefenwasserentnahme im heutigen Zeitpunkt nur einen bescheidenen Erfolg versprechen würde. Als einzige Massnahme, die sonst nicht erfassbaren Nährstoffe in die Hand zu bekommen, wird man sie trotzdem im Auge behalten müssen.

\section{Schlussbemerkungen}

Das letzte Beispiel bekräftigt die Forderung, dass in Seen vom nährstoffarmen Typus eine Nährstoffelimination so weit getrieben werden muss, als es überhaupt möglich ist, und dass die notwendigen Regenentlastungen so gestaltet werden, dass sie nicht direkt in den See, sondern über Rückhaltebecken entlasten.

Die Fragen der Wirtschaftlichkeit der einzelnen Anlagen, so gewichtig sie für den Bauherrn, bzw. für die verantwortliche Behörde auch sein mögen, dürfen dabei nicht den Ausschlag geben. Der vom Menschen belastete und überlastete See reagiert nicht auf das menschliche Ökonomiedenken, nur auf die Nährstoffe. Deshalb ist es, um dies hier zu wiederholen, verfehlt, eine unvollständige Elimination anzustreben, einfach weil es so billiger zu stehen kommt oder eher Gnade findet, wenn die technologischen Mittel eine höhere Wirkung erlauben würden. Dass mit allen besprochenen Massnahmen die Einzugsgebiete von Seen höhere Lasten auferlegt bekommen als reine Flussgebiete, ist eine unabänderliche Tatsache. Einen See rein 
zu erhalten, liegt indessen nicht nur im unmittelbaren Interesse seiner nächsten Umgebung, sondern eines ganzen Landesteils, weshalb diese Mehrlasten durch entsprechende Beihilfen zu unterstützen sind. Dass im weiteren ein gewisser Anteil der zufliessenden Nährstoffe nicht erfassbar ist, ist kein Freipass zur Resignation, im Gegenteil. Das Übel, und es handelt sich tatsächlich um ein grosses und folgenschweres, muss dort angefasst werden, wo es sich anfassen lässt.

Ein Mittel dieser Art ist es, dafür zu sorgen, dass weniger Phosphate überhaupt ins Abwasser gelangen. Lange Zeit hat man sich mit den Ubelständen befasst, welche die Detergentien in den Gewässern verursachen, und erfreulicherweise haben sich die Hersteller der Waschmittel der nötigen Einsicht nicht verschlossen. Bereits werden Waschmittel angeboten, welche in den Kläranlagen abgebaut werden können. Die modernen Waschmittel enthalten aber neben waschaktiven Stoffen auch einen grossen Anteil an Phosphaten. Daneben ist es bekannt, dass der Anstieg des Phosphorgehaltes der Abwässer zu einem guten Teil auf die Phosphatzustätze in den Waschmitteln zurückgeht [I4,37,4I]. Die Wirkung der Phosphate ist, soweit wir das heute beurteilen können, in den Seen unseres Landes wesentlich nachhaltiger, bleibender und folgenschwerer als jene der Detergentien, welche hauptsächlich in den Fliessgewässern zur Geltung kommen. Es ist deshalb am Platze, an den betreffenden Industriezweig die dringende Bitte zu richten, mit dem Zusatz von Pbospborverbindungen zu den Wascbmitteln zurïckzubalten. Mit einer sparsameren Dosierung würde ein aktiver Beitrag an den Schutz der Seen vor Utberdüngung geleistet.

\section{Zusammenfassung}

Stehendes und fliessendes Wasser unterscheidet sich in manchen Belangen grundsätzlich, vor allem im Bestand und in der Bau- und Lebensweise seiner Organismen; dies ist einer der wichtigsten Gründe dafür, dass die Fragen der Überdüngung besonders in stehenden Gewässern von Bedeutung sind. Hier bilden die anorganischen Nährstoffe jenen Faktor, der das Wachstum der pflanzlichen Planktonorganismen steuert. Bei kräftiger Entwicklung des Planktons, der Folge zu starker Düngung, entsteht in der Seetiefe Sauerstoffschwund, es entstehen reduzierende Bedingungen, welche die Rückwanderung von bereits im Sediment festgelegten Nährstoffen ins Wasser fördern. - Während man über die Nährstoffverhältnisse mancher eutropher Seen der Schweiz gut Bescheid weiss, sind Schwierigkeiten insbesondere in der Bestimmung des Phosphors daran schuld, dass unsere 
Kenntnisse über die nährstoffarmen Seen noch dürftig sind. An der EAWAG wurde in letzter Zeit eine verbesserte Methodik der P-Bestimmung ausgearbeitet, welche nun interessante Ergebnisse liefert.

Wir befassen uns im weiteren nur mit dem Phosphor. Im eutrophen See liegt zu Beginn des Seejahres, homogen verteilt, ein grosser Vorrat dieses Nährstoffes vor. In den oberen Schichten wird er durch Algen rasch aufgezehrt; trotzdem steht das Algenwachstum, wie es nach dem «Gesetz des Minimums» nun der Fall sein müsste, nicht still. Die Nachlieferung des Phosphors geht nun im «kurzgeschlossenen Kreislauf» weiter, d. h. die absterbenden Organismen geben bereits in der oberen Wasserschicht so viel Phosphor wieder frei, dass das Wachstum nicht aufhört. Im nährstoffarmen See ist dieser Kreislauf viel ausgeprägter. Phosphoranalysen im Vierwaldstättersee lassen zwar eine Anreicherung in der Tiefe feststellen, doch nimmt der Gehalt an Gesamt-P im Mittel in der oberen, belebten Schicht das Jahr über nicht ab, sondern zeigt nur bemerkenswert starke Schwankungen im Gehalt an anorganischem Phosphat und an gelöstem und ungelöstem organischem Phosphor. Offenbar ist dieser Phosphor dauernd in einem raschen Umsatz begriffen und gelangt gar nicht aus der belebten (trophogenen) Schicht heraus. Daneben können natürlich auch noch weitere, weniger bekannte Faktoren, z. B. Spurenelemente, eine Rolle spielen.

Aus diesen Beobachtungen wird abgeleitet, dass eine Fernhaltung des Phosphors vom See, und namentlich von Seen, welche noch nicht überdüngt sind, nicht auf halbe Massnahmen beschränkt werden darf, sondern dass es angesicht's der immensen Bedeutung dieser Seen als Trinkwasserspeicher notwendig ist, so viel Phosphate zu entfernen oder fernzuhalten, als es überhaupt möglich ist, ohne vorerst auf die wirtschaftlichen Belange zu achten. Die Tatsache, dass ein bedeutender Teil der gesamten Nährstoffzufuhr zu den Seen nicht erfasst werden kann, nämlich jener Teil, der natürlicherweise aus dem Kulturboden und aus der landwirtschaftlichen Düngung stammt, ist kein Grund dafür, die Anforderungen an die Elimination der Phosphate aus dem Abwasser oder an ihre völlige Fernhaltung weniger streng zu gestalten.

Es wird überhaupt empfohlen, in Seeregionen das Abwasserproblem nach Möglichkeit durch den Bau von Ringleitungen, also durch völliges Fernhalten des Abwassers, zu lösen und die Frage der Regenentlastungen, welche einen beträchtlichen Teil des jährlich anfallenden Abwassers ungereinigt dem See zuführen, z. B. mit Rückhaltebecken zu mildern. Die chemische Phosphorelimination ist überall dort am Platze, wo keine Ringleitung erstellt werden kann, also im gesamten Hinterland der Seen. 
Im Gegensatz zu eutrophen Seen verspricht ein Absaugen des mit Phosphor angereicherten Tiefenwassers in nährstoffarmen Seen vorerst wenig Erfolg, weil die wirklich mit Nährstoffen angereicherte Schicht zuwenig mächtig ist und die Menge Nährstoffe, welche überhaupt abgeführt werden könnte, im Verhältnis zum natürlichen Wegtransport durch den Seezufluss zu gering ist.

Die Fernhaltung, bzw. Elimination, ist somit dasjenige Mittel, das die grösste Phosphormenge zu erfassen verspricht. Da die Erhöhung der PKonzentration im Abwasser nicht zuletzt auf die massiven Phosphatzusätze in den Waschmitteln zurückgeht, würde eine zurückhaltendere Dosierung dieser Zusätze durch die Waschmittelindustrie einen positiven Beitrag zur Reinhaltung der Seen bedeuten.

\section{LITERATURVERZEICHNIS}

[I] Wat. \& Wat. Engng. 66, I Io (1962).

[2] Int. Gewässerschutzkommission für den Bodensee, Bericht Nr. I: Zustand und neuere Entwicklung des Bodensees (1963).

[3] AмBÜHL, H., Schweiz. Z. Hydrol. 22, 563 (1960).

[4] BACHOFEN, R., Diss., Univ. Zürich (I960).

[s] Baldinger, F., Schweiz. Z. Hydrol. 19, I8 (1957).

[6] BenorT, R. J., Limnol. \& Oceanogr. 2, 233 (I957).

[7] Coughlin, F. J., J. Amer. Wat. Wks. Ass. 55, 369 (1963).

[8] EINSEle, W., Arch. Hydrobiol. 33, 361 (1938).

[9] EINSELE, W., Arch. Hydrobiol. 39, 664 (1944).

[IO] ELSTER, J., Naturwissenschaften 49, 3, 49 (I962).

[II] FiechTER, R. H., GWF 104, I389 (I963).

[I2] Goltermann, H. L., Acta Bot. Neerl. 9, I (I960).

[13] HaNisCH, B., GWF'104, I399 (1963).

[14] Huber, L., Münch. Beiträge 9, 276 (Oldenbourg-Verlag, München 1962).

[rs] Hutchinson, G. E., A Treatise on Limnology, I (New York 1957).

[I6] JAAG, O., Beseitigung und Reinigung industrieller Abwässer (Oldenbourg-Verlag, München 1959).

[I7] KLIFFMÜLLER, R., Int. Revue ges. Hydrobiol. 47, I, II8 (1962).

[18] Kovalski, V. V., und Letunova, S. V., Zool. Z. 40, 809 (I96I), Biol. Abstr. 41 1353 (1963).

[19] Minder, L., Schweiz. Z. Hydrol. 3, I (1926).

[20] OHLE, W., Naturwissenschaften 40, I53 (I953).

[2I] Pavoni, M., Schweiz. Z. Hydrol. 25, 219 (1963).

[22] Pomeroy, L. R., Mathews, H. M., und MrN, H. S., Limnol. und Oceanogr. 8, So (1963).

[23] RIGLER, F. H., Ecology 37, 550 (1959).

[24] SChelske, C. L., Hooper, F. F., und Haertr, E. J., Ecology 43, 646 (1962). 
[25] Schlapfer, H., Schweiz. Techn. Z. Nr. 28, I2, Juli 1962.

[26] SPRING, W., GWF 104, 40 (1963).

[27] STAUB, R., Schweiz. Z. Hydrol. 23, 82 (196I).

[28] Thomas, E. A., Schweiz. Fischereiztg. (1944), 7/8.

[29] Thomas, E. A., und MärkI, E., Verh. Int. Ver. Limnol. 10, 476 (1949).

[30] Thomas, E. A., Schweiz. Z. Hydrol. 12, I (1950).

[3I] Thomas, E. A., Monatsbulletin SVGW (I953), 2/3.

[32] Thomas, E. A., Mem. Ist. Ital. Idrobiol., suppl. 8, 357 (1955).

[33] THOMAs, E. A., Monatsbull. SVGW (195s), Ir9.

[34] Thomas, E. A., Jb. v. Zürichsee, S. I73 (Stäfa I956/57).

[35] ThOmas, E. A., Monatsbulletin SVGW 43, 6/7 (1963).

[36] VishNiAC, H. S., und RILEY, G. A., Limnol. und Oceanogr. 6, 36 (1962).

[37] Voss, W., GWF 104, 397 (1963).

[38] Watt, W. D., und HaYes, F. R., Limnol. und Oceanogr. 8, 2, 276 (1963).

[39] WILDr, P., Hoch- u. 'Tiefbau, Zürich, 22. 3. I963.

[40] ZigerLI, P., Strasse u. Verkehr (1938).

[4I] ZimmermanN, P., Schweiz. Z. Hydrol. 23, 342 (I96I). 